\title{
Cardioprotective effect of rosuvastatin against isoproterenol-induced myocardial infarction injury in rats
}

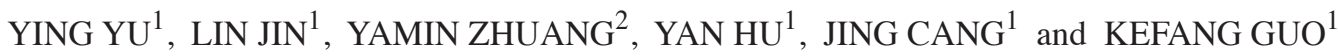 \\ Departments of ${ }^{1}$ Anesthesiology and ${ }^{2}$ Critical Care Medicine, Zhongshan Hospital, \\ Fudan University, Shanghai 200032, P.R. China
}

Received September 27, 2016; Accepted January 25, 2018

DOI: $10.3892 /$ ijmm.2018.3572

\begin{abstract}
Rosuvastatin, a member of 3-hydroxy-3-methylglutaryl coenzyme A reductase inhibitors, exerts various pharmacological activities. This study evaluated the cardioprotective effect of rosuvastatin on isoproterenol-induced myocardial infarction injury in rats. A rat model of myocardial infarction injury was induced by isoproterenol (ISO) for 2 consecutive days, rosuvastatin was administered for 8 weeks. The levels of myocardial infarct size, aspartate transaminase (AST), alanine transaminase (ALT), creatine kinase-MB (CK-MB), lactate dehydrogenase (LDH) activities, as well as malondialdehyde (MDA) levels, superoxide dismutase (SOD), glutathione peroxidase (GPX), catalase (CAT) activities and reduced glutathione (GSH) concentrations were determined. Hematoxylin and eosin staining was used to observe cardiac histological changes. Interleukin-1 $\beta$ (IL-1 $\beta$ ) and IL-18 levels in heart tissues were detected with ELISA kits. The mRNA and protein levels of NOD-like receptor superfamily, pyrin domain containing 3 (NLRP3) inflammasome were measured by qRT-PCR and western blot analysis, respectively. Our results showed that treatment with rosuvastatin reduced myocardial infract area, ameliorated histopathological alterations in myocardium, and decreased activities of myocardial injury marker enzymes in ISO-induced rats. In addition, rosuvastatin remarkably restored ISO-induced elevation of lipid peroxidation and decrease of antioxidants, significantly reduced myocardial pro-inflammatory cytokines concentrations in this animal model. Furthermore, rosuvastatin significantly inhibited the activation of NLRP3 inflammasome in this animal model. This study demonstrates that rosuvastatin significantly alleviates ISO-induced myocardial infarction injury. The mechanism is associated with attenuation of oxidative stress and inflammation, via the inhibition of NLRP3 inflammasome.
\end{abstract}

Correspondence to: Dr Kefang Guo, Department of Anesthesiology, Zhongshan Hospital, Fudan University, 180 Fenglin Road, Shanghai 200032, P.R. China

E-mail: guo.kefang@hotmail.com

Key words: rosuvastatin, myocardial injury, oxidative stress, inflammation, NOD-like receptor superfamily, pyrin domain containing 3 inflammasome

\section{Introduction}

Myocardial infarction is a major form of ischemic heart disease defined as imbalance ischemia and myocardial necrosis $(1,2)$. Even though prognosis has improved substantially over the past decade, acute myocardial infarction remains the most severe manifestation of coronary artery disease, affecting more than 7 million individuals worldwide, accounting for more than 4 million deaths in Europe and Northern Asia every year $(3,4)$. It has been well characterized that oxidative stress and inflammation are the main pathophysiological processes involved in myocardial infarction $(5,6)$. Evidence is accumulating that antioxidant therapy has a potential to prevent ISO-induced myocardial injury (7-9) and myocardial ischemia/reperfusion $(\mathrm{I} / \mathrm{R})$ injury $(10,11)$. Accordingly, NOD-like receptor superfamily, pyrin domain containing 3 (NLRP3) inflammasome is implicated in cellular inflammation processes in response to oxidative stress (12). This inflammasome is protein complex containing NLRP3, ASC and caspase-1. Once the NLRP3 inflammasome is activated, it stimulates caspase-1 activation, which in turn promotes the processing and secre-

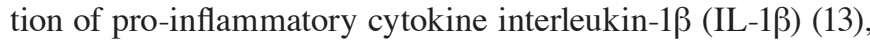
which has been implicated to play a role in I/R injury. Reactive oxygen species (ROS) have been identified as an important NLRP3 inflammasome activator in various diseases, such as hepatic (14), and renal I/R injury (15). Moreover, previous studies have demonstrated that NLRP3 inflammasome was activated in myocardial I/R injury in cardiac microvascular endothelial cells (16), cardiac fibroblasts (17), which indicates a role of NLRP3 inflammasome in the development of myocardial injury.

Rosuvastatin, a member of 3-hydroxy-3-methylglutaryl coenzyme A reductase inhibitors, exerts various pharmacological activities, such as anti-inflammatory $(18,19)$, anti-oxidative $(20,21)$, cardioprotective activities $(22,23)$. Previous studies have reported that rosuvastatin inhibited neuronal cell apoptosis and improved neurological deficit in transient middle cerebral artery occlusion (tMCAO)/reperfusion injury (24), and promoted angiogenesis in myocardial infarct rats (25). However, the mechanisms by which rosuvastatin protects against myocardial injury are still incompletely understood. Furthermore, rosuvastatin alleviates diabetic cardiomyopathy by suppressing the cardiac NLRP3 inflammasome activation and IL-1 $\beta$ production in a type 2 diabetes rat 
model (26). Importantly, rosuvastatin treatment significantly decreases NLRP3 expression, and its downstream cytokines in peripheral blood monocytes of acute myocardial infarction patients and unstable angina patients (27), suggest that rosuvastatin may ameliorate myocardial infarction injury via NLRP3 inflammasome.

Based on the above, the present study investigated the cardioprotective effect of rosuvastatin on ISO-induced myocardial injury in rats, focusing on the antioxidant and anti-inflammatory role, and elucidated whether the cardioprotective effect of rosuvastatin in ISO-induced myocardial injury is mediated by NLRP3 inflammasome.

\section{Materials and methods}

Materials and chemicals. Isoproterenol hydrochloride was purchased from Sigma Chemical Co. (St. Louis, MO, USA). Rosuvastatin was kindly provided by AstraZeneca (Shanghai, China). The aspartate transaminase (AST), alanine transaminase (ALT), creatine kinase (CK-MB), and lactate dehydrogenase (LDH) kits were procured from Nanjing Jiancheng Bioengineering Institute (Jiangsu, China). Superoxide dismutase (SOD), catalase (CAT) and glutathione peroxidase (GPX) activities as well as reduced glutathione (GSH) and malondialdehyde (MDA) levels in heart tissues were measured by commercially available kits (Beyotime Institute of Biotechnology, Haimen, China). Commercially ELISA kits for IL-1 $\beta$ and IL-18 were obtained from R\&D Systems (Minneapolis, MN, USA). Antibodies of NLRP3 and ASC were obtained from Cell Signaling Technology (Danvers, MA, USA), antibodies of caspase-1 and glyceraldehyde 3-phosphate dehydrogenase (GAPDH) were purchased from Santa Cruz Biotechnology, Inc. (Santa Cruz, CA, USA). All other chemicals and reagents used in this study were of analytical grade.

Animals. Forty-five male Wistar-Albino rats (180-200 g) were purchased from the Experimental Animal Center of Fudan University (Shanghai, China). The animals were maintained under standard laboratory conditions at $25 \pm 2^{\circ} \mathrm{C}$ and $50 \pm 15 \%$ humidity with an alternate $12 \mathrm{~h}$ cycle of light and dark. They were acclimatized to the conditions of the animal house for 1 week before the experiment and allowed free access to standard laboratory diet and water ad libitum. All animal procedures were done in accordance with the guidelines for the care and use of laboratory animals approved by the Ethics Committee for Animal Experimentation of Fudan University.

Experimental protocol. Rats were randomly divided into two groups, NC group $(n=9)$ and ISO group $(n=36)$. A rat model of myocardial ischemia was induced by subcutaneous injection of ISO hydrochloride for 2 consecutive days, while NC group were injected with normal saline for 2 consecutive days. After one week, ISO group were randomly divided into four subgroups: Model group: rats received $1 \mathrm{ml} / \mathrm{kg} /$ day $1 \%$ Tween-80 suspension in distilled water by oral gavage for 8 consecutive weeks, RSV5, RSV10 and RSV15 group: rats received rosuvastatin $(5,10$ or $15 \mathrm{mg} / \mathrm{kg}$, respectively) in distilled water by oral gavage for 8 consecutive weeks. The dose of ISO and RSV was selected based upon previous studies $(24,28,29)$. After the end of the animal experiment, rats were anesthetized and sacrificed, blood samples were collected and centrifuged to obtain serum for the biochemical assays.

Histopathological studies. After blood collection, the heart tissues were rapidly removed, then the cardiac apex was immediately fixed in $4 \%$ paraformaldehyde, processed in ethanol and embedded in paraffin wax. The cardiac apex were stained with hematoxylin and eosin (H\&E) and examined under a light microscope (Olympus, Tokyo, Japan).

Determination of pro-inflammatory cytokines in heart. Enzyme immunoassay of IL-1 $\beta$ and IL-18 in heart homogenate was performed by using ELISA kits according to manufacturer's instructions (R\&D Systems). The color intensity was read at $450 \mathrm{~nm}$ with a microplate reader (Tecan Ltd., Mannedorf, Switzerland) and the cytokines levels were expressed as $\mathrm{pg} / \mathrm{mg}$ of tissue.

Measurement of MI markers in the serum. The serum was used to assay AST, ALT, CK-MB and LDH activities. The activities of AST, ALT, CK-MB and LDH were assayed using commercial kits purchased from Jiancheng Bioengineering Institute (Nanjing, China) according to the manufacturer's instructions.

Evaluation of lipid peroxidation and antioxidant enzyme levels. After experimental treatment, the homogenates of heart tissues were centrifuged at $16,000 \mathrm{rpm}$ for $10 \mathrm{~min}$. The supernatant was used to assay MDA levels, and SOD, CAT, GPX activities, as well as GSH concentrations according to the manufacturer's instructions, on a microplate reader at 560 and $532 \mathrm{~nm}$. The commercially available assay kits were purchased from Jiancheng Bioengineering Institute.

$q R T-P C R$. The mRNA expression levels of NLRP3, ASC and caspase-1 were analyzed via qRT-PCR; total RNA sample from rat heart tissues was extracted and purified using TRIzol reagent (Invitrogen, Carlsbad, CA, USA) following the manufacturer's instructions. Total RNA was then reverse transcribed into cDNA with an M-MLV reverse transcriptase kits according to the manufacturer's protocol. Following the reverse transcription, qPCR was performed to quantify the RNA levels of NLRP3, ASC and caspase-1 using SYBR-Green Supermix (Bio-Rad, Hercules, CA, USA) and the data were analyzed using the $2^{-\Delta \Delta C t}$ method. GAPDH was used as a housekeeping gene for mRNA analysis. The primer sequences are listed in Table I.

Western blot analysis. Total protein was loaded per well, resolution on a $10 \%$ sodium dodecyl sulfate polyacrylamide gel (SDS-PAGE), and then transferred onto a polyvinylidene difluoride membrane (Millipore, Bedford, MA, USA). The membrane was then blocked with 5\% skim milk and subsequently incubated with primary antibodies for NLRP3 $(1: 1,000)$, ASC $(1: 1,000)$ and caspase-1 antibodies $(1: 500)$ overnight at $4^{\circ} \mathrm{C}$. Membranes were subsequently incubated with appropriate HRP-conjugated secondary antibody at room temperature for $1 \mathrm{~h}$. Immunoreactive bands were visualized via enhanced chemiluminescence (Millipore) and quantified via densitometry using ImageJ (National Institutes of Health). 
Table I. RT-PCR primer sequences.

\begin{tabular}{lll}
\hline Gene & \multicolumn{1}{c}{ Forward } & \multicolumn{1}{c}{ Reverse } \\
\hline NLRP3 & 5'-CAGCGATCAACAGGCGAGAC-3' & 5'-AGAGATATCCCAGCAAACCTATCCA-3' \\
ASC & 5'-TTATGGAAGAGTCTGGAGCT-3' & 5'-CAGCTGATGGACCTGACTGA-3' \\
Caspase-1 & 5'-CGTGGAGAGAAACAAGGAGTG-3' & 5'-AATGAAAAGTGAGCCCCTGAC-3' \\
GAPDH & 5'-TTCAACGGCACAGTCAAGG-3' & 5'-CACCAGTGGATGCAGGGAT-3' \\
\hline
\end{tabular}

NLRP3, NOD-like receptor superfamily, pyrin domain containing 3; GAPDH, glyceraldehyde 3-phosphate dehydrogenase.
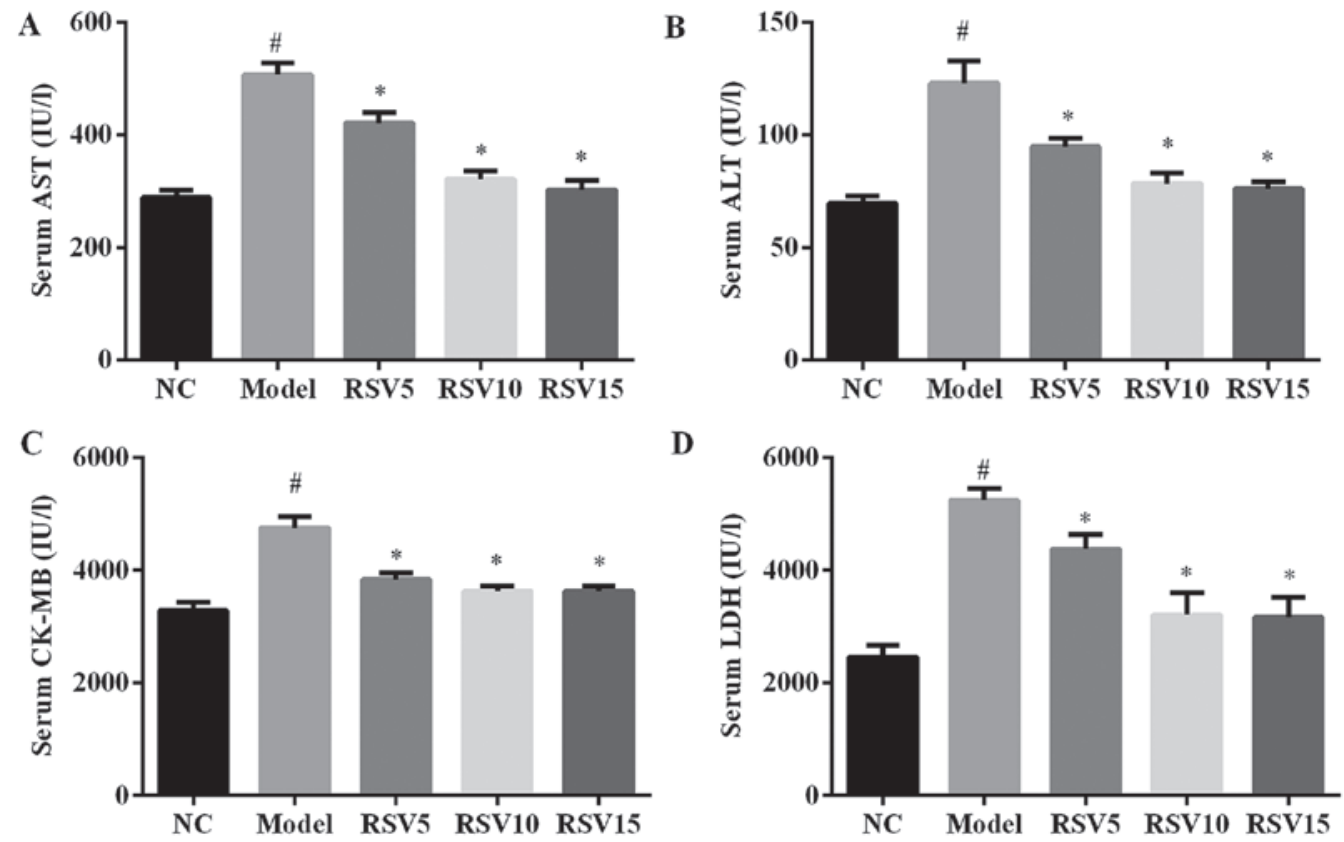

Figure 1. (A) Serum aspartate transaminase (AST), (B) alanine transaminase (ALT), (C) creatine kinase-MB (CK-MB) and (D) lactate dehydrogenase (LDH) levels between the groups. Results are expressed as mean $\pm \mathrm{SD}, \mathrm{n}=9$. NC, normal control group; model, isoproterenol group; RSV, rosuvastatin group. ${ }^{*} \mathrm{P}<0.05$ compared with normal control group; ${ }^{*} \mathrm{P}<0.05$ compared with model group.

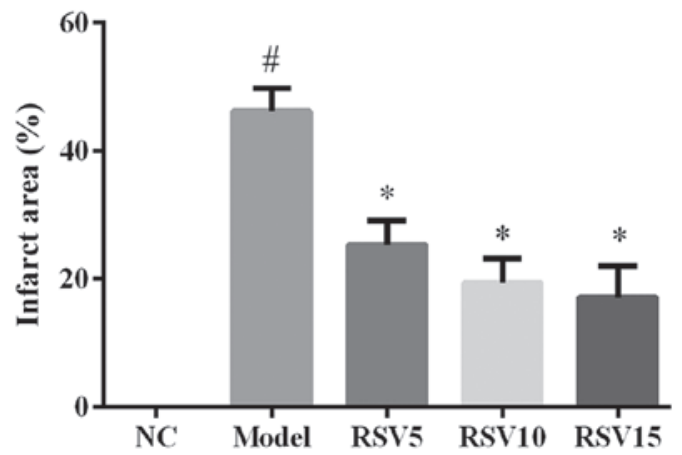

Figure 2. Representative histological photographs of myocardial tissue between the groups. NC, normal control group; model, isoproterenol group; RSV, rosuvastatin treated group.

Statistical analysis. All data were expressed as mean $\pm \mathrm{SD}$, and analyzed using one-way ANOVA followed by a post-hoc test to determine the statistical difference between groups. Statistical analysis was performed using the GraphPad Prism 5 (GraphPad Software, Inc., San Diego, CA, USA). A value of $\mathrm{P}<0.05$ was considered statistically significant.

\section{Results}

Effect of rosuvastatin on AST, ALT, CK-MB and LDH enzyme activities in ISO induced rats. To investigate whether rosuvastatin could ameliorate ISO-induced myocardial injury, we analyzed the myocardial injury marker activities in heart tissue of all groups. As illustrated in Fig. 1, two subcutaneous injections of ISO significantly induces cardiac dysfunction as evidenced by greatly increase serum activities of AST, ALT, $\mathrm{CK}-\mathrm{MB}$ and $\mathrm{LDH}$ in comparison to normal control group $(\mathrm{P}<0.05)$. However, by administration of rosuvastatin, serum AST, ALT, CK-BA and LDH activities were obviously relieved in this animal model $(\mathrm{P}<0.05)$.

Effect of rosuvastatin on histopathological assessments in heart in ISO induced rats. Fig. 2 shows that ISO induced a significant infarction area in comparison to the normal control group $(\mathrm{P}<0.05)$. Compared with the model group, myocardial infarction area of rosuvastatin treated groups were remarkably diminished $(\mathrm{P}<0.05)$ (Fig. 2). Furthermore, results of histopathological examination also confirmed the protective effect of rosuvastatin in ISO-induced myocardial injury. As 

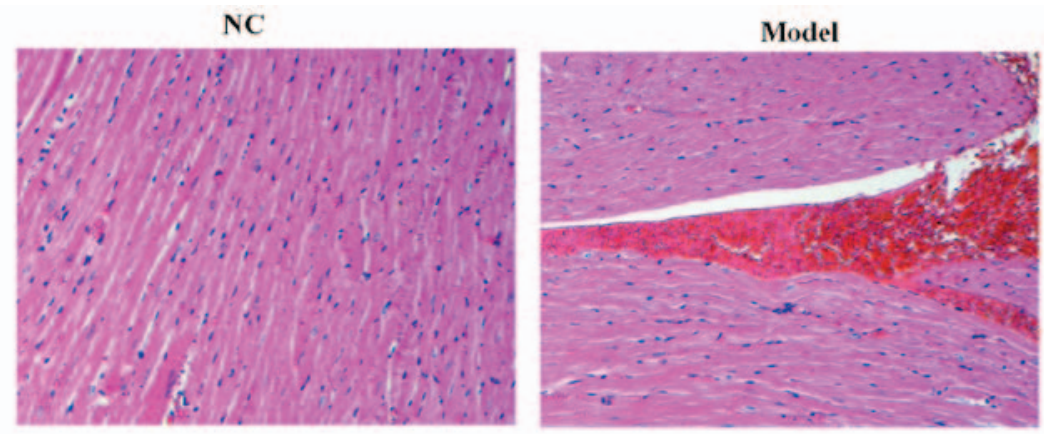

RSV5

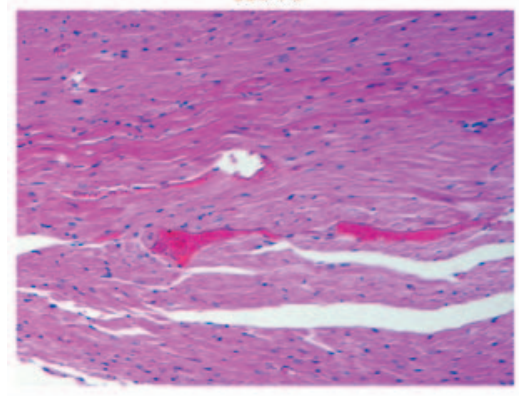

RSV10

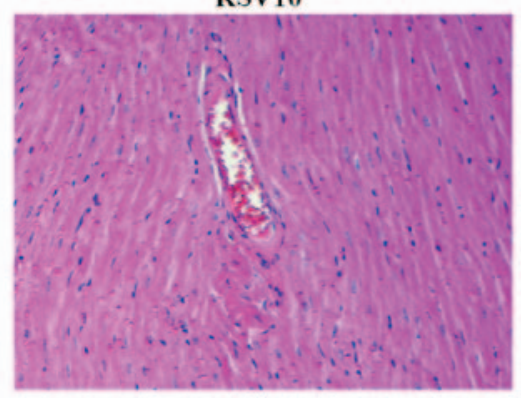

RSV15

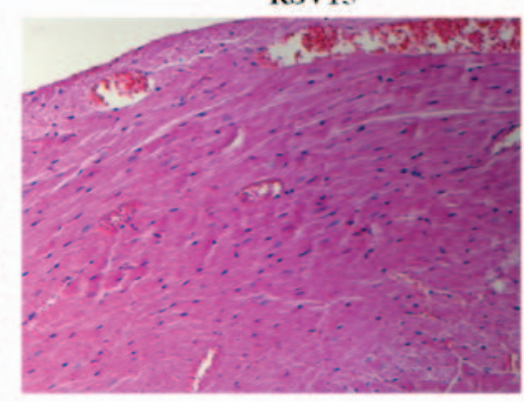

Figure 3. Effect of rosuvastatin on myocardial infarct area. Results are expressed as mean $\pm \mathrm{SD}, \mathrm{n}=9$. NC, normal control group; model, isoproterenol group; RSV, rosuvastatin group; ${ }^{\text {P }}<0.05$ compared with normal control group; ${ }^{\mathrm{P}}<0.05$ compared with model group.

illustrated in Fig. 3, NC group showed a normal histoarchitecture, while obvious necrosis of myofibers with cell infiltration, as well as extravasations of red blood cells were observed in the heart tissue of ISO rats (Fig. 3). Importantly, rosuvastatin significantly ameliorated these changes in ISO rats.

Effect of rosuvastatin on lipid peroxidation and oxidative stress parameters in ISO induced rats. Then, we investigated the effect of rosuvastatin on ISO-induced lipid peroxidation and oxidative stress in rats. As shown in Fig. 4, marked increase of MDA, a lipid peroxidation byproduct, was observed in the heart of ISO group. Moreover, rats administered ISO showed decreased activities of antioxidants such as SOD, CAT and GPX, downregulated non-enzymatic antioxidant (GSH) concentrations, as compared to normal control rats. However, rosuvastatin significantly reduced cardiac MDA levels, increased SOD, CAT and GPX activities and GSH concentrations in ISO-treated rats $(\mathrm{P}<0.05)$. These results suggest that ISO injection causes oxidative stress in heart of rats, which is suppressed by the treatment of rosuvastatin.

Effect of rosuvastatin on pro-inflammatory cytokines in ISO induced rats. The production of pro-inflammatory cytokines such as IL-1 $\beta$ and IL-18 in heart tissues are shown in Fig. 5. Compared to normal control group, injection of ISO significantly increased the secretion levels of IL-1 $\beta$ and IL-18 in the heart $(\mathrm{P}<0.01)$. Treatment with rosuvastatin reduced ISO-induced elevation of cardiac IL-1 $\beta$ and IL-18 secretion in this animal model.

Effect of rosuvastatin on NLRP3 inflammasome activation in ISO induced rats. In order to evaluate whether rosuvastatin alleviated ISO-induced myocardial injury via NLRP3 inflammasome, the mRNA and protein expression levels of
NLRP3, ASC and caspase-1 in the heart of all experimental groups were detected. ISO injection obviously induced the activation of NLRP3, characterized by significantly increased cardiac mRNA (Fig. 6) and protein (Fig. 7) expression levels of NLRP3, ASC and caspase-1 $(\mathrm{P}<0.05)$. Rosuvastatin markedly decreased NLRP3, ASC and caspase-1 at both mRNA and protein levels.

\section{Discussion}

In this study, we confirmed the cardioprotective effects of rosuvastatin against ISO-induced myocardial infarction injury in rats. Treatment with rosuvastatin significantly reduced myocardial infract area, improved myocardial histoarchitecture, and decreased serum levels of myocyte marker enzymes in ISO-induced myocardial injury in rats. In addition, rosuvastatin remarkably restored ISO-induced elevation of antioxidants and decreased lipid peroxidation.

ISO, a synthetic non selective $\beta$-adrenergic agonist, ISO-induced myocardial injury has been widely used to investigate the effect of drugs on myocardial infraction $(30,31)$. In the present study, a rat model of myocardial injury was successfully established, as evidenced by dramatically increased serum levels of AST, ALT, CK-MB and LDH, and abnormal cardiac microstructure observed on histopathological examination. These results are in line with previous in vivo studies $(9,32)$. Furthermore, the present study further confirmed that rosuvastatin can ameliorate ISO-induced myocardial infarction injury, evidenced by dramatically decreasing serum levels of myocardial injury markers and obviously diminished histopathological alterations.

There is a relationship between oxidative stress and myocardial infraction injury (33). Disturbance in oxidants and antioxidant metabolism has been noted in patients with 
A

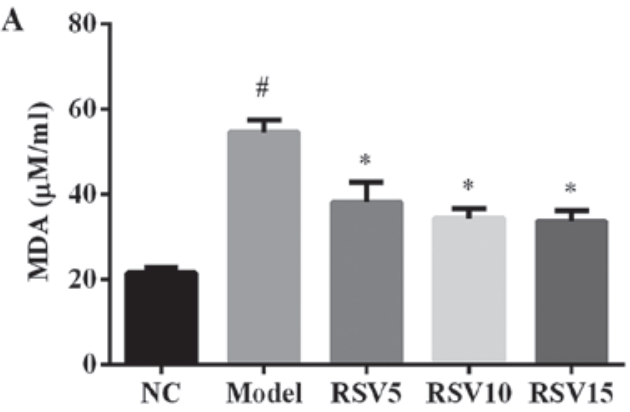

C

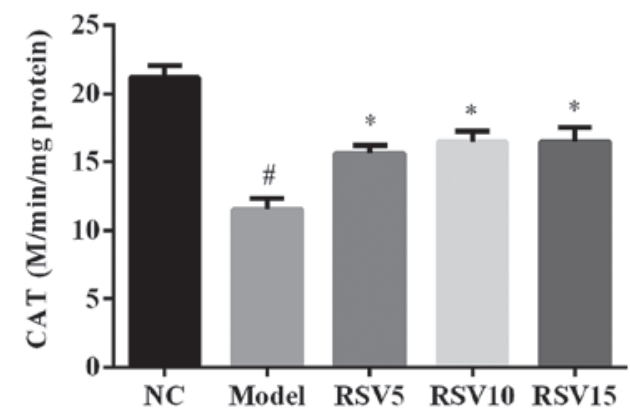

E

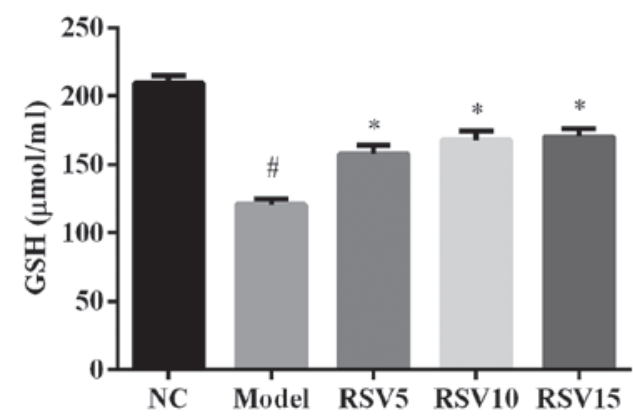

$\mathbf{B}$

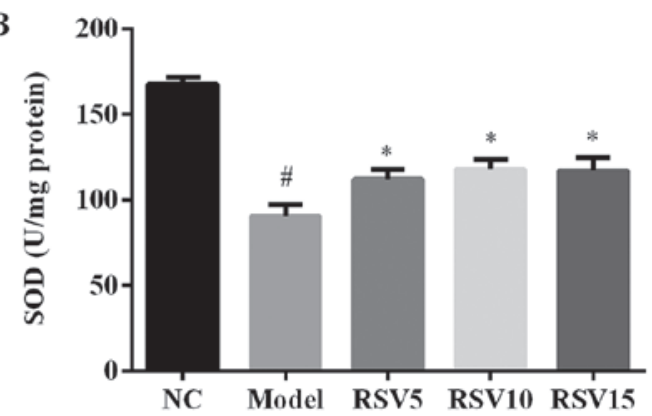

D

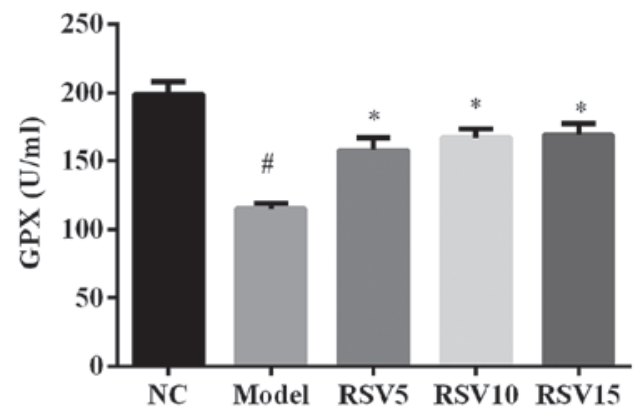

Figure 4. (A) Cardiac malondialdehyde (MDA) levels, (B) superoxide dismutase (SOD), (C) catalase (CAT), (D) glutathione peroxidase (GPX) activities, and (E) glutathione $(\mathrm{GSH})$ concentrations between the groups. Results are expressed as mean $\pm \mathrm{SD}, \mathrm{n}=9$. Activity is expressed as $\mathrm{U} / \mathrm{mg}$ protein for SOD, $\mu$ mol of $\mathrm{H}_{2} \mathrm{O}_{2}$ decomposed/second/mg protein for CAT, $\mu \mathrm{mol}$ of $\mathrm{GSH}$, oxidized/min/mg of protein for GPX. NC, normal control group; model, isoproterenol group; RSV, rosuvastatin group. ${ }^{\#} \mathrm{P}<0.05$ compared with normal control group; ${ }^{*} \mathrm{P}<0.05$ compared with the model group.
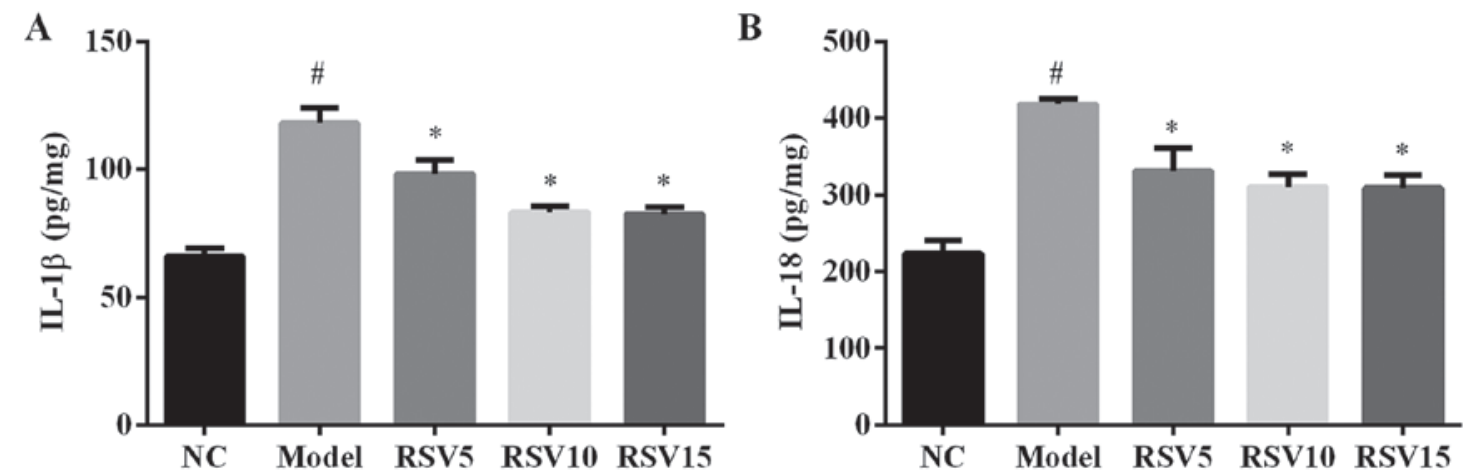

Figure 5. (A) Cardiac interleukin-1 $\beta$ (IL-1 $\beta$ ) and (B) IL-18 production levels between the groups. Results are expressed as mean \pm SD, $n=9$. NC, normal control group; model, isoproterenol group; RSV, rosuvastatin group. " $\mathrm{P}<0.05$ compared with normal control group; "P<0.05 compared with model group.

acute myocardial infraction $(34,35)$. Free radical scavenging enzymes, such as CAT, SOD and GPX, GSH are first line cellular defense against oxidative damage (36). This study observed increased MDA and decreased antioxidants such as SOD, CAT, GPX and GSH in ISO-treated rat heart tissues.
Thus, it suggests that oxidative stress may be involved in ISO-induced myocardial infraction injury of rats. However, the treatment of rosuvastatin remarkably restored ISO-induced oxidative stress by increasing antioxidants activities and decreasing lipid peroxidation. 

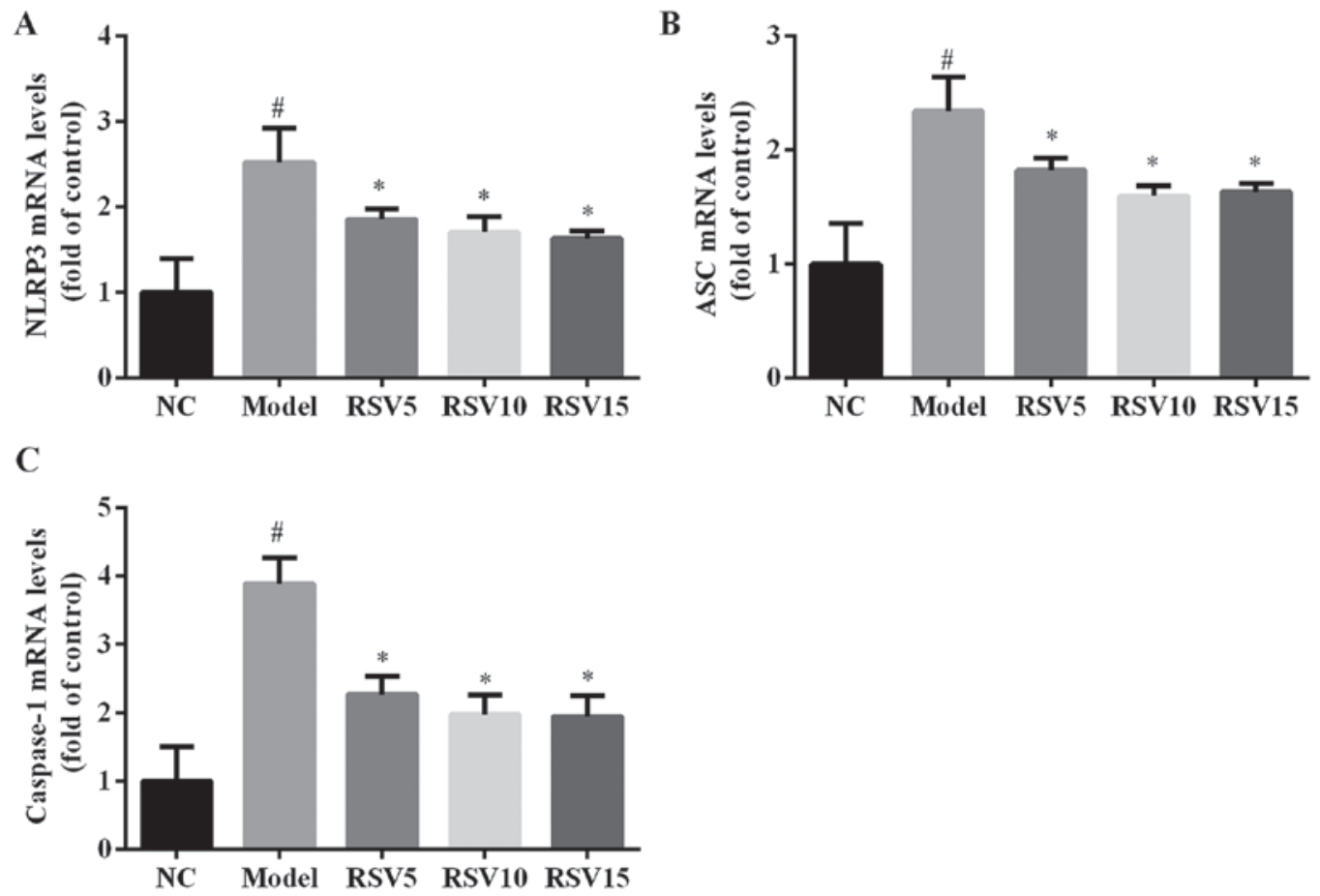

Figure 6. Cardiac NOD-like receptor superfamily, (A) pyrin domain containing 3 (NLRP3), (B) ASC and (C) caspase-1 mRNA levels between all groups Results are expressed as mean $\pm \mathrm{SD}, \mathrm{n}=9$. NC, normal control group; model, isoproterenol group; RSV, rosuvastatin group. "P $<0.05$ compared with normal control group; ${ }^{*} \mathrm{P}<0.05$ compared with model group.

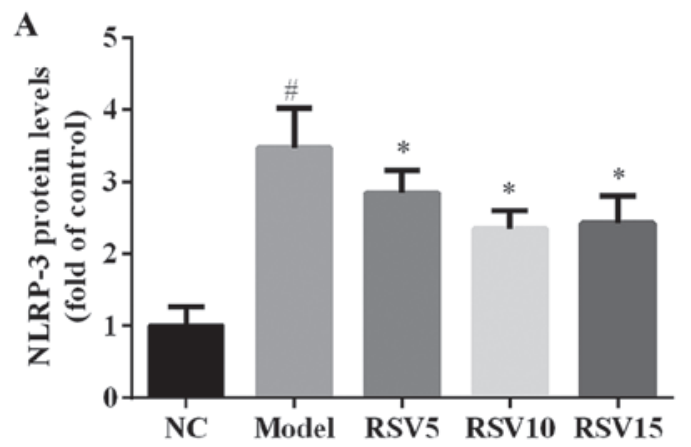

C

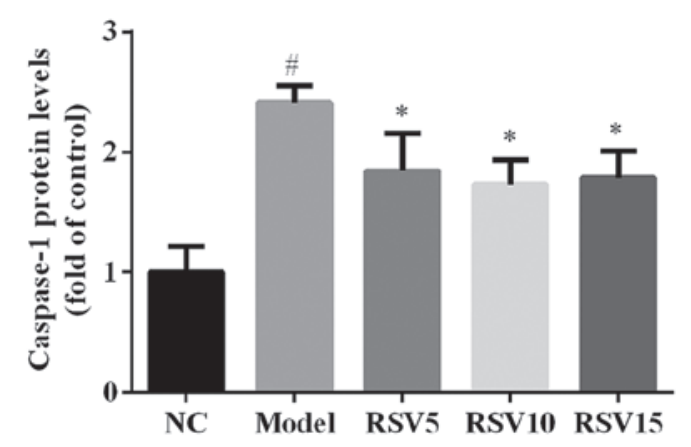

B

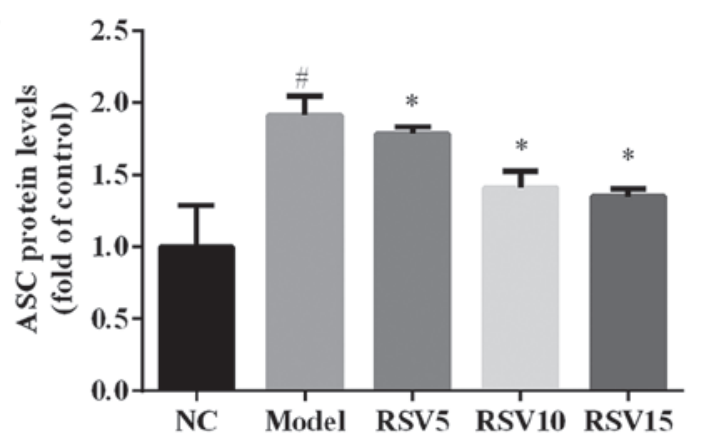

D
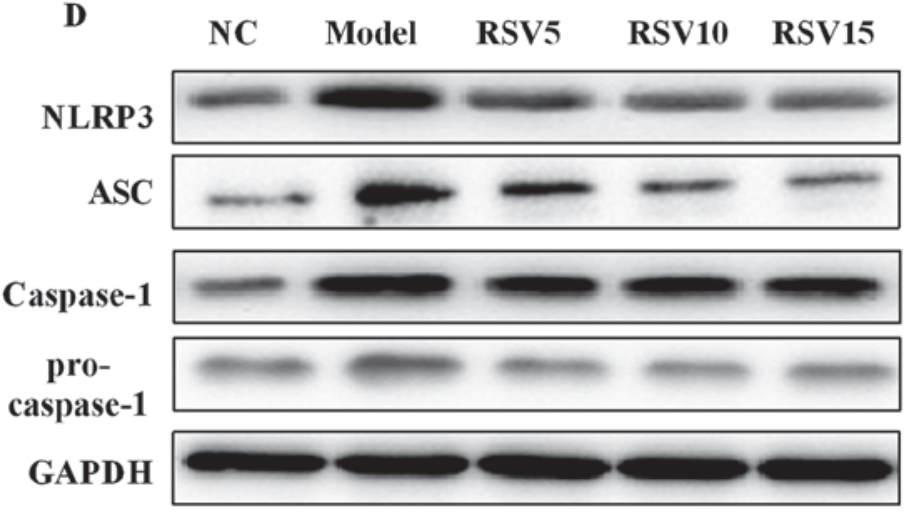

Figure 7. (A-D) Cardiac NLRP3, ASC and caspase-1 protein levels between the groups. Results are expressed as mean \pm SD, $n=9$. NC, normal control group; model, isoproterenol group; RSV, rosuvastatin group. ${ }^{\#} \mathrm{P}<0.05$ compared with normal control group; ${ }^{*} \mathrm{P}<0.05$ compared with model group.

NLRP3 inflammasome is implicated in cellular inflammation processes in response to oxidative stress (12). Activated NLRP3 inflammasome has been observed in the peripheralblood monocytes of patients with acute myocardial infarction $(27,37)$.
Moreover, overproduction of pro-inflammatory cytokine IL-1 $\beta$ and IL-18 is involved in I/R injury (38), which is critically dependent on the activation of NLRP3 inflammasome. In the present study, our results confirmed that NLRP3 inflammasome 
activated in the heart of ISO-induced myocardial injury, as measured by cardiac NLRP3, ASC and caspase-1 expression levels. Clinical study also has demonstrated that patients with acute myocardial infarction show a significant increase of IL-1 $\beta$ plasma levels (39). Accordingly, our study found that ISO remarkably increased IL-1 $\beta$ and IL-18 production in the heart tissue. Furthermore, Liu et al reported that NLRP3 siRNA and BAY 11-7082 significantly ameliorated myocardial I/R (16). Collectively, these findings imply a role of NLRP3 inflammasome in myocardial infarction injury, and inhibiting NLRP3 inflammasome activation may be a novel therapeutic target for the treatment of myocardial infarction injury.

Rosuvastatin, is an approved drug for treating patients with hyperlipidemia and hypercholesterolemia. Given that rosuvastatin exerts both anti-inflammatory $(18,19)$ and antioxidative $(20,21)$ effect, we studied the effect of rosuvastatin on ISO-induced myocardial infarction injury. We found that the treatment of rosuvastatin remarkably restored ISO-induced by increasing antioxidant activities and decreasing lipid peroxidation, significantly reducing cardiac pro-inflammatory cytokines production. Importantly, a previous study indicated that rosuvastatin significantly downregulated NLRP3 expression, and its downstream cytokines in peripheral blood monocytes of acute myocardial infarction patients (27). Consistently, our results found that rosuvastatin remarkably decreased NLRP3, ASC and caspase-1 mRNA and protein levels in the heart of ISO rats implying that rosuvastatin inhibited cardiac NLRP3 inflammasome activation in ISO rats. Therefore, we suggest that rosuvastatin alleviates ISO-induced myocardial infarction injury by attenuating oxidative stress and via the inhibition of NLRP3 inflammasome.

In conclusion, the present study showed that rosuvastatin significantly alleviated ISO-induced myocardial infarction injury in rats. The effect is associated with attenuation of oxidative stress and inflammation, via the inhibition of NLRP3 inflammasome. However, further studies are needed to explore the exact mechanism by which rosuvastatin inhibits the activation of NLRP3 inflammasome in the heart tissue of myocardial infraction injury. The results of this study suggest that the cholesterol-lowering medicine rosuvastatin may have potential for the prevention and treatment of myocardial infarction.

\section{Acknowledgements}

Not applicable.

\section{Funding}

No funding was received.

\section{Availability of data and material}

The datasets used and/or analyzed during the current study are available from the corresponding author on reasonable request.

\section{Authors' contributions}

YY and KG conceived and designed the study. LJ, YZ and YH performed the experiments. JC analyzed the data and wrote the paper. All authors read and approved the final manuscript.

\section{Ethics approval and consent to participate}

All animal procedures were done in accordance with the guidelines for the care and use of laboratory animals approved by the Ethics Committee for Animal Experimentation of Fudan University.

\section{Consent for publication}

Not applicable.

\section{Competing interests}

The authors declare that they have no competing interests.

\section{References}

1. Eltzschig HK and Eckle T: Ischemia and reperfusion - from mechanism to translation. Nat Med 17: 1391-1401, 2011.

2. Whellan DJ: Heart failure disease management: Implementation and outcomes. Cardiol Rev 13: 231-239, 2005.

3. Nichols M, Townsend N, Scarborough P and Rayner M: Cardiovascular disease in Europe 2014: Epidemiological update. Eur Heart J 35: 2929-2933, 2014.

4. Reed GW, Rossi JE and Cannon CP: Acute myocardial infarction. Lancet 389: 197-210, 2017.

5. Sawyer DB, Siwik DA, Xiao L, Pimentel DR, Singh K and Colucci WS: Role of oxidative stress in myocardial hypertrophy and failure. J Mol Cell Cardiol 34: 379-388, 2002.

6. Neri M, Fineschi V, Di Paolo M, Pomara C, Riezzo I, Turillazzi E and Cerretani D: Cardiac oxidative stress and inflammatory cytokines response after myocardial infarction. Curr Vasc Pharmacol 13: 26-36, 2015.

7. Kumar M, Kasala ER, Bodduluru LN, Dahiya V and Lahkar M: Baicalein protects isoproterenol induced myocardial ischemic injury in male Wistar rats by mitigating oxidative stress and inflammation. Inflamm Res 65: 613-622, 2016.

8. Chen M, Wang M, Yang Q, Wang M, Wang Z, Zhu Y, Zhang Y, Wang C, Jia Y, Li Y, et al: Antioxidant effects of hydroxysafflor yellow $\mathrm{A}$ and acetyl-11-keto- $\beta$-boswellic acid in combination on isoproterenol-induced myocardial injury in rats. Int J Mol Med 37: 1501-1510, 2016.

9. Li H, Xie YH, Yang Q, Wang SW, Zhang BL, Wang JB, Cao W, Bi LL, Sun JY, Miao S, et al: Cardioprotective effect of paeonol and danshensu combination on isoproterenol-induced myocardial injury in rats. PLoS One 7: e48872, 2012.

10. Suchal K, Bhatia J, Malik S, Malhotra RK, Gamad N, Goyal S, Nag TC, Arya DS and Ojha S: Seabuckthorn pulp oil protects against myocardial ischemia-reperfusion injury in rats through activation of Akt/eNOS. Front Pharmacol 7: 155, 2016.

11. Yang M, Chen J, Zhao J and Meng M: Etanercept attenuates myocardial ischemia/reperfusion injury by decreasing inflammation and oxidative stress. PLoS One 9: e108024, 2014.

12. Abderrazak A, Syrovets T, Couchie D, El Hadri K, Friguet B, Simmet T and Rouis M: NLRP3 inflammasome: From a danger signal sensor to a regulatory node of oxidative stress and inflammatory diseases. Redox Biol 4: 296-307, 2015.

13. Ogura Y, Sutterwala FS and Flavell RA: The inflammasome: First line of the immune response to cell stress. Cell 126: 659-662, 2006.

14. Zhu P, Duan L, Chen J, Xiong A, Xu Q, Zhang H, Zheng F, Tan Z, Gong F and Fang M: Gene silencing of NALP3 protects against liver ischemia-reperfusion injury in mice. Hum Gene Ther 22: 853-864, 2011.

15. Shigeoka AA, Mueller JL, Kambo A, Mathison JC, King AJ, Hall WF, Correia JS, Ulevitch RJ, Hoffman HM and McKay DB: An inflammasome-independent role for epithelial-expressed Nlrp3 in renal ischemia-reperfusion injury. J Immunol 185: 6277-6285, 2010.

16. Liu Y, Lian K, Zhang L, Wang R, Yi F, Gao C, Xin C, Zhu D, Li Y, Yan W, et al: TXNIP mediates NLRP3 inflammasome activation in cardiac microvascular endothelial cells as a novel mechanism in myocardial ischemia/reperfusion injury. Basic Res Cardiol 109: 415, 2014. 
17. Kawaguchi M, Takahashi M, Hata T, Kashima Y, Usui F, Morimoto H, Izawa A, Takahashi Y, Masumoto J, Koyama J, et al: Inflammasome activation of cardiac fibroblasts is essential for myocardial ischemia/reperfusion injury. Circulation 123: 594-604, 2011.

18. Monetti M, Canavesi M, Camera M, Parente R, Paoletti R, Tremoli E, Corsini A and Bellosta S: Rosuvastatin displays anti-atherothrombotic and anti-inflammatory properties in apoE-deficient mice. Pharmacol Res 55: 441-449, 2007.

19. Funderburg N, Clagett B, Jiang Y, Debanne S, Storer N, Labbato D, Juchnowski S, Ferrari B, Lederman M and McComsey GA: Rosuvastatin reduces immune activation and inflammation in treated HIV infection. Conference on Retroviruses and Opportunistic Infections. Boston, MA, 2014. Poster 335.

20. Duarte T, da Cruz IB, Barbisan F, Capelleto D, Moresco RN and Duarte MM: The effects of rosuvastatin on lipid-lowering, inflammatory, antioxidant and fibrinolytics blood biomarkers are influenced by Val16Ala superoxide dismutase manganesedependent gene polymorphism. Pharmacogenomics J 16 501-506, 2016.

21. Liang D, Zhang Q, Yang H, Zhang R, Yan W, Gao H, Wang J, Zhang X, Chen Y and Cao F: Anti-oxidative stress effect of loading-dose rosuvastatin prior to percutaneous coronary intervention in patients with acute coronary syndrome: A prospective randomized controlled clinical trial. Clin Drug Investig 34 773-781, 2014.

22. Ke D, Fang J, Fan L, Chen Z and Chen L: Regulatory T cells contribute to rosuvastatin-induced cardioprotection against ischemia-reperfusion injury. Coron Artery Dis 24: 334-341, 2013.

23. Chen Z, Cao Y, Qian J, Ma J, Zou Y and Ge J: Cardioprotection of rosuvastatin against cardiac dysfunction after coronary microembolization via alleviating inflammatory induced microinfarctions. Circulation 132: A13434, 2015.

24. Ma M, Uekawa K, Hasegawa $Y$, Nakagawa T, Katayama T, Sueta D, Toyama K, Kataoka K, Koibuchi N, Kuratsu J, et al: Pretreatment with rosuvastatin protects against focal cerebral ischemia/reperfusion injury in rats through attenuation of oxidative stress and inflammation. Brain Res 1519: 87-94, 2013.

25. Zaitone SA and Abo-Gresha NM: Rosuvastatin promotes angiogenesis and reverses isoproterenol-induced acute myocardial infarction in rats: Role of iNOS and VEGF. Eur J Pharmacol 691: 134-142, 2012.

26. Luo B, Li B, Wang W, Liu X, Liu X, Xia Y, Zhang C, Zhang Y, Zhang $M$ and An F: Rosuvastatin alleviates diabetic cardiomyopathy by inhibiting NLRP3 inflammasome and MAPK pathways in a type 2 diabetes rat model. Cardiovasc Drugs Ther 28: 33-43, 2014.
27. Altaf A, Qu P, Zhao Y, Wang H, Lou D and Niu N: NLRP3 inflammasome in peripheral blood monocytes of acute coronary syndrome patients and its relationship with statins. Coron Artery Dis 26: 409-421, 2015.

28. Kumar M, Kasala ER, Bodduluru LN, Dahiya V, Sharma D, Kumar V and Lahkar M: Animal models of myocardial infarction: Mainstay in clinical translation. Regul Toxicol Pharmacol 76: 221-230, 2016.

29. Han S, Cai W, Yang X, Jia Y, Zheng Z, Wang H, Li J, Li Y, Gao J, Fan L, et al: ROS-mediated NLRP3 inflammasome activity is essential for burn-induced acute lung injury. Mediators Inflamm 2015: 720457, 2015.

30. Karthikeyan K, Bai BR and Devaraj SN: Cardioprotective effect of grape seed proanthocyanidins on isoproterenol-induced myocardial injury in rats. Int J Cardiol 115: 326-333, 2007.

31. Abbas AM: Cardioprotective effect of resveratrol analogue isorhapontigenin versus omega-3 fatty acids in isoproterenolinduced myocardial infarction in rats. J Physiol Biochem 72: 469-484, 2016

32. Geng ZH, Huang L, Song MB and Song YM: Protective effect of a polysaccharide from Salvia miltiorrhiza on isoproterenol (ISO)-induced myocardial injury in rats. Carbohydr Polym 132: 638-642, 2015.

33. Hill MF and Singal PK: Antioxidant and oxidative stress changes during heart failure subsequent to myocardial infarction in rats. Am J Pathol 148: 291-300, 1996.

34. Madole MB, Bachewar NP and Aiyar CM: Study of oxidants and antioxidants in patients of acute myocardial infarction. Adv Biomed Res 4: 241, 2015.

35. Patil N, Chavan V and Karnik ND: Antioxidant status in patients with acute myocardial infarction. Indian J Clin Biochem 22: 45-51, 2007.

36. Wattanapitayakul SK and Bauer JA: Oxidative pathways in cardiovascular disease: Roles, mechanisms, and therapeutic implications. Pharmacol Ther 89: 187-206, 2001.

37. Sandanger $\varnothing$, Ranheim T, Vinge LE, Bliksøen M, Alfsnes K, Finsen AV, Dahl CP, Askevold ET, Florholmen G, Christensen G, et al: The NLRP3 inflammasome is up-regulated in cardiac fibroblasts and mediates myocardial ischaemia-reperfusion injury. Cardiovasc Res 99: 164-174, 2013.

38. Pomerantz BJ, Reznikov LL, Harken AH and Dinarello CA: Inhibition of caspase 1 reduces human myocardial ischemic dysfunction via inhibition of IL-18 and IL-1beta. Proc Natl Acad Sci USA 98: 2871-2876, 2001.

39. Guillén I, Blanes M, Gómez-Lechón MJ and Castell JV: Cytokine signaling during myocardial infarction: Sequential appearance of IL-1 beta and IL-6. Am J Physiol 269: R229-R235, 1995. 